\title{
The multigenic structure of the MHC locus contributes to positive selection efficiency: A role for MHC class II gene- specific restriction
}

\author{
Miguel Caetano Monteiro, Sofia Couceiro and Carlos Penha-Gonçalves \\ Instituto Gulbenkian de Ciência, Oeiras, Portugal
}

The study of $\mathrm{T}$ cell positive selection in the thymus has long been focused on the specificity of the MHC-TCR interactions, making use of genetically manipulated mice that display TCR specificities or selecting peptides of limited diversity. However, little is known on the role of the MHC molecules irrespective of the peptide specificity and the implications of MHC multigenic structure in thymic positive selection have not been addressed. Here, we investigated the effect of MHC class II genetic configuration on the positive selection efficiency of naturally generated pre-selection repertoires in the mouse thymus. Analysis of positively selected thymocyte populations in MHC-congenic and -transgenic mice revealed that expression of I-E molecule in the thymic cortex increases positive selection efficiency of CD4 cells by approximately $50 \%$. We show that increments in positive selection attributable to either the I-A and I-E genes are not due to increased MHC class II expression in the thymic cortex and are not affected by the number of MHC alleles. Collectively, our findings imply that MHC class II generestricted TCR specificities significantly contribute to positive selection efficiency, introducing the notion that multigenic structure of the MHC locus serves to increase selection of non-overlapping TCR repertoires.

Received 26/6/05

Revised 18/8/05

Accepted 29/9/05

[DOI 10.1002/eji.200535190]

Key words:

MHC $\cdot$ Multigenic - Repertoire

development

Thymic selection

\section{Introduction}

The cellular and the molecular events involved in thymic selection of developing $\mathrm{T}$ cells have been extensively described. It is well established that mainstream $\mathrm{T}$ cell development is critically dependent on binding the $\mathrm{T}$ cell receptor (TCR) of immature T cells to peptideloaded major histocompatibility complex (MHC) molecules expressed on different cell types harbored in the thymus. Thus, TCR-MHC binding drives interactions between $\mathrm{CD} 4^{+} \mathrm{CD} 8^{+} \mathrm{TCR} \alpha \beta$ thymocytes and epithelial cells in the thymic cortex, which in turn lead to the delivery of a signal for survival and maturation, a process known as positive selection [1]. Analysis of different experimental mouse models led to the general

Correspondence: Carlos Penha-Gonçalves, Instituto Gulbenkian de Ciência, Rua Quinta Grande 6, 2768 Oeiras, Portugal Fax: +351 214407970

e-mail: cpenha@igc.gulbenkian.pt

Abbreviation: DP: $\mathrm{CD} 4^{+} \mathrm{CD} 8^{+}$double-positive notion that the strength of TCR-MHC interactions governs thymic selection. Thus, TCR ligands inducing positive selection would have lower affinity/avidity than ligands inducing negative selection or $\mathrm{T}$ cell activation in the periphery [2-4].

In line with this rationale, much of the research on thymocyte positive selection has been concerned with the specificity of the interaction between TCR and MHCbound peptides during positive selection. Subsequently, this led to a debate on the requirement of a diverse set of positively selecting peptides for the generation of the selected $\mathrm{T}$ cell repertoire diversity [5]. While single MHC/peptide combinations were shown to select TCR with different antigen specificities, the collected evidence suggests that positive selection makes use of a diverse set of peptides that has low occupancy on surface MHC molecules but contributes significantly to the diversity of the selected TCR repertoire $[2,6,7]$.

Studies in transgenic systems have indicated that variations in thymic density of MHC-restricting molecules did not affect the ability to positively select high 
numbers of cells carrying high-affinity TCR [8-10]. In contrast, a recent report shows that in a low-affinity transgenic TCR system, efficiency of positive selection decreases when the density of MHC class I-restricting molecules is reduced [11]. Moreover, it was reported that positive selection efficiency of a transgenic TCR is limited by competition for the TCR ligand, represented by the cognate MHC-peptide complex [12] . These lines of evidence support the notion that selection efficiency of a given TCR is a function of the probability of maintaining enduring TCR-MHC interactions, which in turn can be controlled by TCR ligand availability as well as by TCR ligand affinity. In vivo microscopy has revealed that the dynamics of thymocyte-cortical epithelium interactions include both relatively short and prolonged contacts. This would be expected if selectable thymocytes scan the thymic epithelium for positively selecting ligands until establishing stable contacts [13].

The study of thymic positive selection efficiency has relied in the analysis of genetically manipulated mice to express limited TCR diversity $[8,14]$. Nevertheless, in non-transgenic systems positive selection acts upon individual TCR that are supposed to occur at rather low frequency while the TCR diversity of selectable cells is expected to be very high. However, there is little information on quantitative aspects of positive selection in non-manipulated animals carrying a naturally generated pre-selection repertoire. As pointed out by von Boehmer et al. [1], most studies related to the role of positively selecting ligands have been concerned with the MHC-bound peptides rather than the MHC molecules irrespective of the peptides. The MHC system introduces TCR ligand diversity by contributing with various genes of each MHC molecule class (multiple genes) and with the numerous alleles of each gene (polymorphism). For instance, the mouse MHC class II molecules are represented by two molecules, I-A and I-E, which means diversity may go up to four different class II $\beta$ chain alleles in one individual. It is expected that the MHC genetic diversity would play a role on TCR repertoire composition, but the impact of the MHC genetic configuration in the efficiency of positive selection has not been studied.

We have previously reported that the size of the thymic $\mathrm{CD}^{+}{ }^{+}$compartment is controlled by the MHC locus [15]. In this study, we analyzed the overall efficiency of positive selection of $\mathrm{CD}^{+}$cells, with naturally generated pre-selection repertoires. To address this question we compared a set of mouse strains expressing either I-E, I-A or I-E plus I-A molecules. It is well described that certain TCR specificities are restricted to particular MHC class II alleles but it is not known what the contributions are of MHC class II gene-restricted specificities to the composition of selected TCR repertoires. Our results introduce the notion that MHC class II gene number is a relevant factor on the overall efficiency of thymic positive selection.

\section{Results}

The size of thymic compartments is controlled by the MHC genotype

We observed previously that the size of the CD4 singlepositive cell compartment was reduced in the thymus of C57BL/6 mice as compared to BALB/c mice. Subsequently, a genetic mapping experiment showed that a

Table 1. Description of the mouse strains ${ }^{a)}$

\begin{tabular}{|c|c|c|c|}
\hline & background strain & MHC haplotype & I-E $\alpha$ thymic expression \\
\hline B6.Cg/NTac-Foxn1 $1^{\text {nu }}$ & C57BL/6 & $\mathrm{b}$ & absent \\
\hline B6.tg $E \alpha^{d}$ & $\mathrm{C} 57 \mathrm{BL} / 6$ & $\mathrm{~b}$ & transgenic \\
\hline B6.tg $E \alpha^{d} . A \beta^{-/-b)}$ & $\mathrm{C} 57 \mathrm{BL} / 6$ & $\mathrm{~b}$ & transgenic \\
\hline C57BL/6 & $\mathrm{C} 57 \mathrm{BL} / 6$ & $b$ & absent \\
\hline B6.C-H2<bd $>$ & $\mathrm{C} 57 \mathrm{BL} / 6$ & $\mathrm{bd}$ & endogenous \\
\hline B6.C-H2 $<\mathrm{d}>/ \mathrm{bBy}$ & $\mathrm{C} 57 \mathrm{BL} / 6$ & $d$ & endogenous \\
\hline C.B10-H2<b $>/$ LilMcd & $\mathrm{BALB} / \mathrm{c}$ & $\mathrm{b}$ & absent \\
\hline C.B10-H2<bd $>$ & $\mathrm{BALB} / \mathrm{c}$ & $\mathrm{bd}$ & endogenous \\
\hline $\mathrm{BALB} / \mathrm{c}$ & $\mathrm{BALB} / \mathrm{c}$ & $d$ & endogenous \\
\hline
\end{tabular}

a) For the described characteristics, the B6.Rag2 ${ }^{-/-}$, B6.Rag2 ${ }^{-/-}$.C-H2<bd $>$and B6.Rag2 ${ }^{-/-}$.C-H2 $<\mathrm{d}>$ mice match the C57BL/6, B6.C$\mathrm{H} 2<\mathrm{bd}>$ and B6.C-H2 $<\mathrm{d}>/$ bBy mice, respectively.

b) The B6.tg $E \alpha^{\mathrm{d}}$. A $\beta^{-/-}$strain results from the introduction of the $A \beta$ null mutation into the B6.tg E $\alpha^{\mathrm{d}}$ strain and expresses the I-E molecule but not the I-A molecule of the MHC class II. 
region on mouse chromosome 17 encompassing the MHC locus controls the frequency of $\mathrm{CD}^{+} \mathrm{CD} 8^{+}$ double-positive (DP) and CD4 single-positive cells in the thymus [15]. In the present study, we started by using MHC-congenic mouse strains to confirm that the MHC haplotype determines variations in the relative size of both mature and immature thymocyte compartments.

The B6.C-H $2<\mathrm{d}>$ congenic strain carries the $\mathrm{MHC}^{\mathrm{d}}$ haplotype on the C57BL/ 6 genetic background while the C.B10-H2 $<$ b $>$ congenic strain carries the $\mathrm{MHC}^{\mathrm{b}}$ haplotype on the BALB/c genetic background (Table 1). The analysis of these mouse strains showed that size variation in the DP and CD4 mature cell compartments is attributable to the MHC region irrespectively of the genetic background (Fig. 1). In fact, mice that carry the
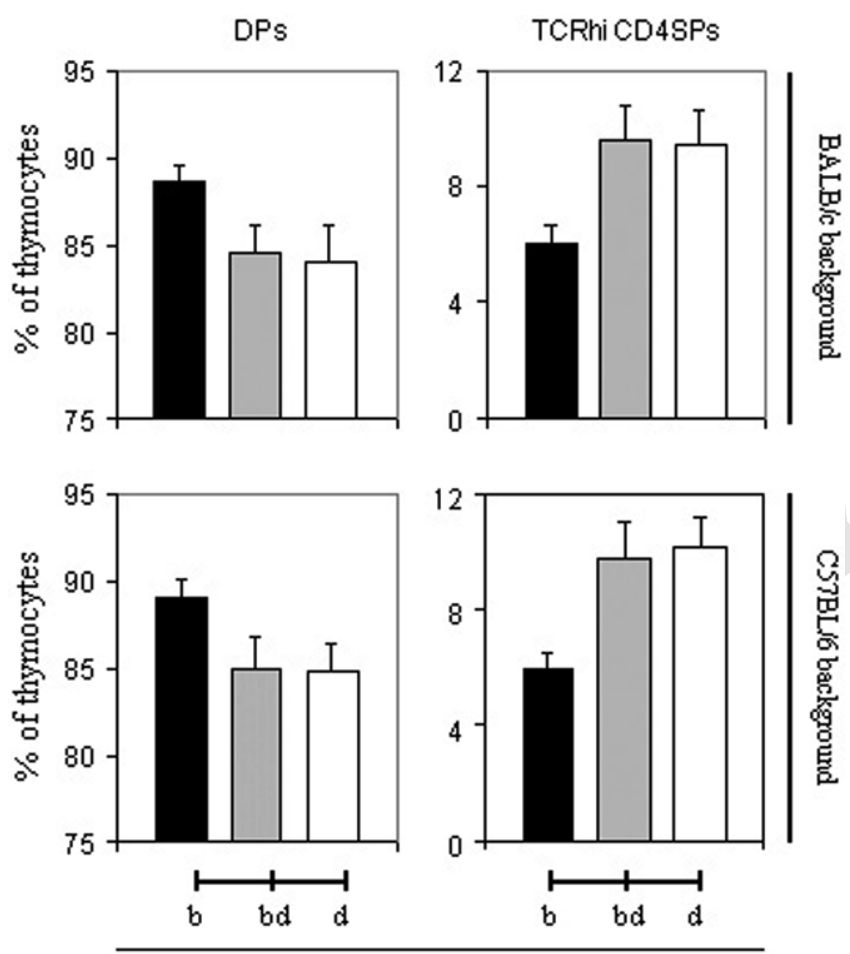

MHC genotype

Figure 1. The MHC genotype controls the size of immature and mature populations in the thymus. Relative sizes of immature DP and both mature CD4 and CD8 compartments were measured in female mice carrying the $\mathrm{MHC}^{\mathrm{b}}$ haplotype (black bars), the $\mathrm{MHC}^{\mathrm{d}}$ haplotype (white bars) and the $\mathrm{MHC}^{\mathrm{b} / \mathrm{d}}$ heterozygous haplotype (gray bars). Analyzed mice carried either the BALB/C or the C57BL/6 genetic background. Mice with the BALB/C genetic background (upper panel) included C.B10$\mathrm{H} 2<\mathrm{b}>\quad(n=10$; black bars), BALB/c ( $n=6$; white bars) and F1(BALB/c $\times$ C.B10-H2 $<b>)$ mice $(n=10$; gray bars). Mice with the $C 57 \mathrm{BL} / 6$ genetic background (lower panel) included C57BL/ $6(n=10$; black bars), B6.C-H2 $<\mathrm{d}>(n=9$; white bars $)$ and F1 (C57BL/6 $\times$ B6.C-H2 $<\mathrm{d}>$ ) mice $(\mathrm{n}=10$; gray bars). Error bars represent standard deviation for each mouse group. Thymocyte subpopulations are expressed as percent of total thymocytes and were identified by flow cytometry using anti-mouse CD4, CD8 and TCR $\beta$ antibodies.
MHC $^{\mathrm{b}}$ genotype (C57BL/ 6 and C.B10-H2 $<$ b $>$ strains) show higher frequency of DP and lower frequency of CD4 cells as compared to mice that share the same genetic background and are homozygous for $\mathrm{MHC}^{\mathrm{d}}$ (B6.C-H $2<\mathrm{d}>$ and BALB/c strains, respectively). Thymic compartments in $\mathrm{MHC}^{\mathrm{d}}$ homozygous and $\mathrm{MHC}^{\mathrm{b} / \mathrm{d}}$ heterozygous mice were undistinguishable, implying that $\mathrm{MHC}^{\mathrm{b}}$ genetic effect on the size of thymocyte subpopulations has a recessive behavior (Fig. 1). The observed differences in the relative sizes of DP and CD4 compartments suggest that the $\mathrm{MHC}^{\mathrm{b}}$ haplotype has lower efficiency in positively selecting immature DP cells into the CD4 mature compartment. In contrast, the size variation of the $\mathrm{CD} 8$ single-positive population in different genetic backgrounds and MHC genotypes did not exceed $2 \%$ of the thymocytes (data not shown).

\section{The MHC genotype controls the efficiency of thymic positive selection}

Next, we analyzed the amount of positively selected cells within the DP compartment across different MHC genotype configurations. To this end we made use of a set of cell surface molecules known to be up-regulated in positively selected but still immature thymocytes, namely CD69, TCR $\alpha \beta$ and CD53 $[16,17]$. Within the same genetic background the mice carrying the $\mathrm{MHC}^{\mathrm{d}}$ haplotype had a higher percentage of DP cell upregulating positive selection markers while showing lower frequency of DP cells with no signs of positive selection (Fig. 2). Conversely, mice homozygous for the $\mathrm{MHC}^{\mathrm{b}}$ haplotype in the genetic background of both C57BL/6 and BALB/c mouse strains showed a lower proportion of positively selected cells within the DP compartment, again indicating that $\mathrm{MHC}^{\mathrm{b}}$ had a quasirecessive behavior. The results show that the effect of the MHC haplotype previously observed at the level of the CD4 compartment size is imposed in the transition between non-selected DP thymocyte stage and positively selected stage within the immature DP compartment. This analysis of MHC-congenic strains reveals that the MHC genotype has a measurable effect on the efficiency of positive selection, a process that is predominantly mediated by the cortical thymic epithelium.

\section{Efficiency of positive selection is governed by thymic epithelial MHC genotype}

To test whether the MHC genotype of the thymic epithelium can control the efficiency of positive selection, we generated a set of bone marrow chimeras that received thymocyte precursors of the $\mathrm{MHC}^{\mathrm{b}}$ haplotype but expressed different MHC genotypes in the thymic epithelia. Thus, mature T cell-deprived bone marrow from C57BL/ 6 nude mice was transferred into 

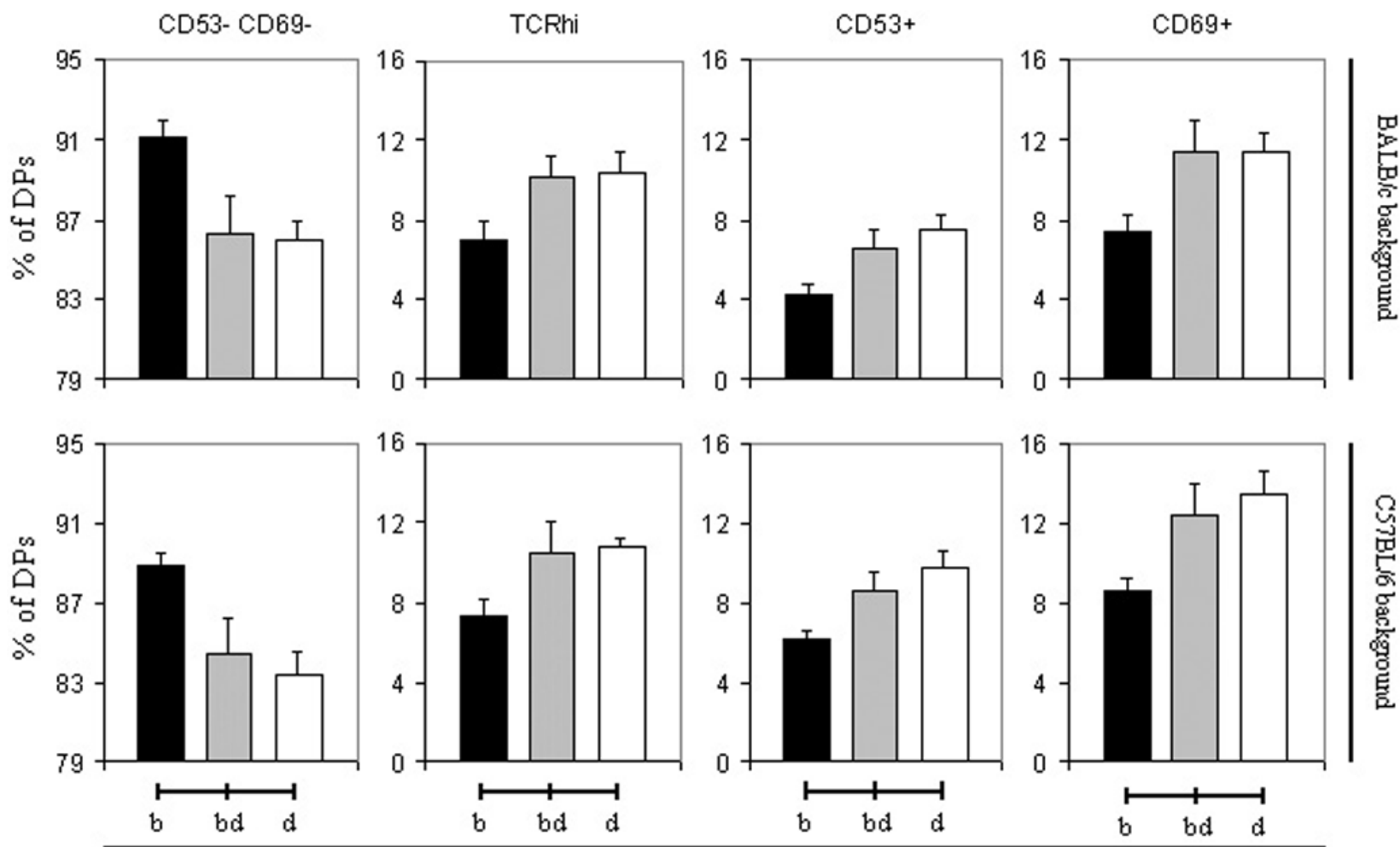

MHC genotype

Figure 2. The MHC genotype controls the frequency of positively selected cells within the DP compartment. The relative sizes of thymocyte subpopulations within the immature DP compartment were measured in the mice represented in Fig. 1. Mouse groups are represented as in Fig. 1 and error bars represent the standard deviation. Thymocyte subpopulations are expressed as percent of the DP population and were identified by flow cytometry using anti-mouse CD4, CD8, CD69 and either CD53 or TCR $\beta$ antibodies.

irradiated lymphocyte deprived RAG-deficient mice carrying different MHC haplotypic configurations on the C57BL/6 genetic background (MHC ${ }^{\mathrm{b}}, \mathrm{MHC}^{\mathrm{d}}$ and $\mathrm{MHC}^{\mathrm{b} / \mathrm{d}}$ ). The size of DP and CD4 populations in the bone marrow chimeras showed to be dependent on the recipient MHC genotype following the trend observed of MHC genetic control in non-chimeric mice (Fig. 3). Moreover, we observed that DP subpopulations carrying positive selection markers had lower frequency in recipient mice that express the $\mathrm{MHC}^{\mathrm{b}}$ haplotype (Fig. 4). These results clearly show that the observed differences in thymic subpopulation size can not be explained by hematopoietic thymic components. Most likely, the control of the positive selection efficiency of CD4 single-positive cells by the MHC genotype is exerted through the expression of MHC class II molecules on thymic epithelial cells.

\section{MHC class II surface expression in the thymus is not influenced by the MHC haplotype}

It is well known that the $\mathrm{MHC}^{\mathrm{b}}$ haplotype lacks the expression of the MHC class II I-E molecule, while the $\mathrm{MHC}^{\mathrm{d}}$ haplotype expresses both the I-E and the I-A MHC class II molecules. Two possibilities can be envisaged
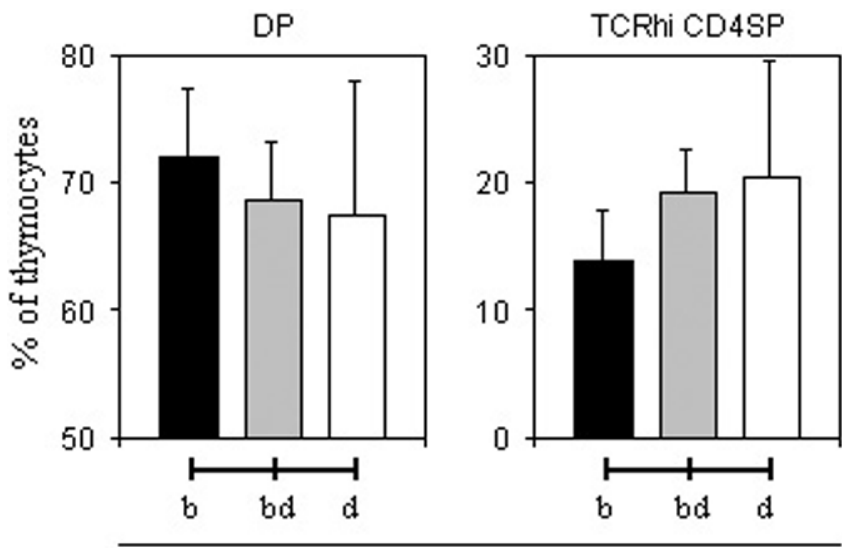

MHC genotype

Figure 3. The size of immature and mature populations in bone marrow chimeras is controlled by the recipient MHC genotype. Relative sizes of immature DP and both mature CD4 and CD8 compartments were measured in chimeric bone marrow recipient mice that received bone marrow cells from $T$ cell deprived nude mice. The recipient mice comprised B6.Rag2 ${ }^{-/}$ ( $n=7$; black bars), B6.Rag2 ${ }^{-1-} . \mathrm{C}-\mathrm{H} 2<\mathrm{bd}>(n=6$; gray bars) and B6.Rag2 ${ }^{-1-}$.C-H2 $<\mathrm{d}>$ mice ( $n=3$; white bars). Error bars represent standard deviation for each mouse group. Thymocyte subpopulations are expressed as percent of total thymocytes and were identified by flow cytometry using anti-mouse CD4, CD8 and TCR $\beta$ antibodies. 

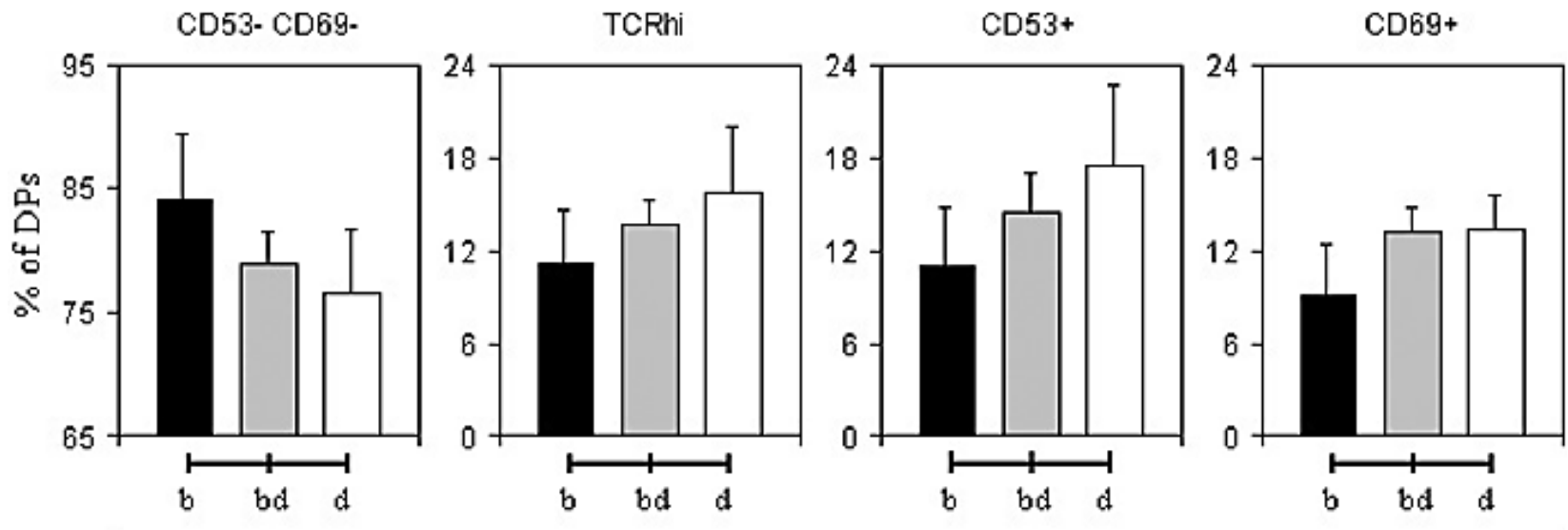

MHC genotype

Figure 4. The frequency of positively selected cells within the DP compartment is controlled by the recipient MHC genotype in bone marrow chimeras. The relative sizes of thymocyte subpopulations within the immature DP compartment were measured in the mice represented in Fig. 3. Flow cytometry was performed using anti-mouse CD4, CD8, CD69 and either CD53 or TCR $\alpha \beta$ antibodies. The error bars represent standard deviation for each mouse group.

through which increased positive selection would be achieved in mice expressing the I-E molecule in the thymus epithelium. On one hand, the presence of I-E molecule in the thymus cortex could enrich the diversity of MHC-peptide selecting combinations. On the other hand, the expression of I-E could increase the density of MHC class II molecules at the surface of the cortical cells, which in turn would increase the opportunities of successful interactions with selectable $\mathrm{CD} 4^{+} \mathrm{CD} 8^{+}$ TCR $\alpha \beta$ thymocytes.

To test whether the expression level of MHC class II molecules was lower in mice with $\mathrm{MHC}^{\mathrm{b}}$ haplotype as compared to mice carrying $\mathrm{MHC}^{\mathrm{d}}$ haplotype, we quantified MHC class II surface expression in the cortex epithelium. Thus, thymic sections from C57BL/6, B6.C $<\mathrm{H} 2 \mathrm{~d}>$ and $\mathrm{BALB} / \mathrm{c}$ mice were immunostained with anti-MHC class II antibodies and fluorescence intensity was recorded and compared (Fig. 5A). Although the patterns of cortical fluorescence were somewhat different, the results show that no significant difference was apparent in the amount of MHC class II molecules expressed in the three strains analyzed (Fig. 5B). These results indicate that the observed differences in positive selection can not be attributed to quantitative differences in cortical expression of MHC class II molecules. Therefore, we hypothesize that the increase in efficiency of positive selection observed when the I-E molecule is expressed could result from the higher diversity of MHC-peptide present on cortical thymic epithelium.
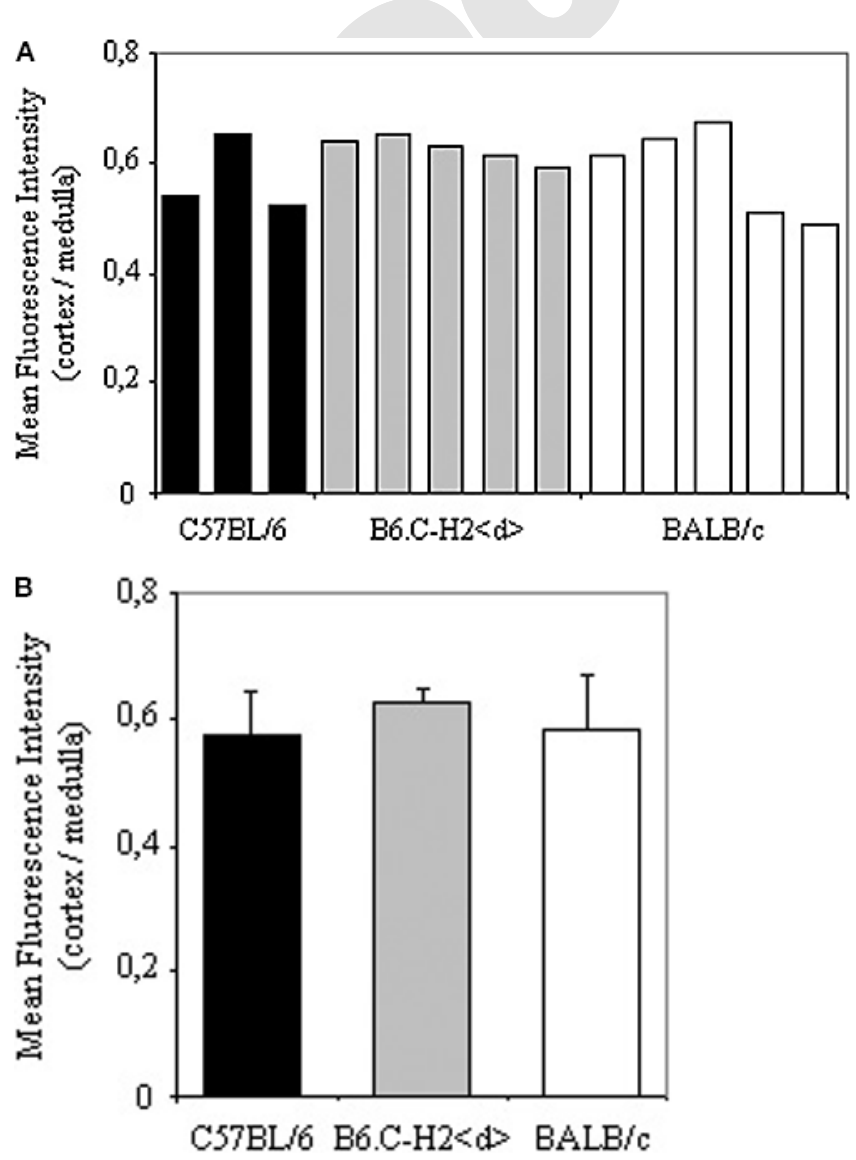

Figure 5. Surface density of cortex MHC class II molecules is not dependent on the MHC genotype. MHC class II expression was measured by immunostaining of thymic sections with a pan-MHC class II antibody. (A) Values of cortex fluorescence intensity expressed as the ratio between cortex and medulla fluorescence calculated for individual mice as described in the Material and methods section. (B) Average values for the groups of mice represented in (A). Black bars represent C57BL/6 $(n=3)$, gray bars B6.C-H2 $<\mathrm{d}>/ \mathrm{bBy}(n=5)$, and white bars BALB/c mice $(n=5)$. 


\section{Multiple MHC class II genes increase efficiency of CD4 cell positive selection}

Next, we investigated whether the number of MHC class II genes expressed in the thymus could explain the observed difference in positive selection efficiency. Thus, we compared thymocyte populations from C57BL/6, B6.tg $E \alpha^{\mathrm{d}} \mathrm{A} \beta^{-/-}$and B6.tg $\mathrm{E} \alpha^{\mathrm{d}}$ mice expressing I-A, I-E and I-A plus I-E molecules on thymic epithelia, respectively [18, 19]. This analysis showed that the sole expression of the I-E molecule in the thymic cortex of an otherwise C57BL/ 6 mouse strain restores the efficiency of positive selection (compare Fig. 1 and 6). In fact, B6.tg E $\alpha^{\mathrm{d}}$ mice have higher levels of CD4 single-positive cells as well as a higher percentage of DP cells expressing positive selection markers than the

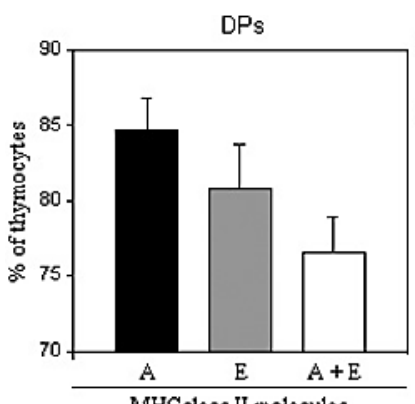

MHCclass II molecules

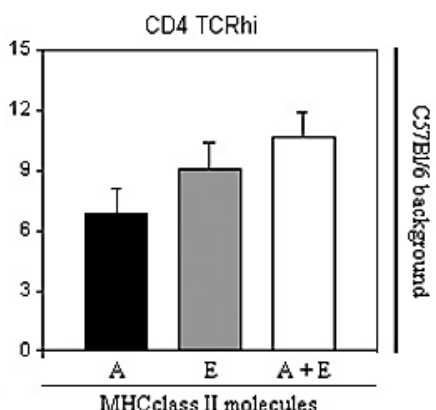

Figure 6. Multiple MHC class II gene expression in the thymic cortex controls the size of immature and mature thymocyte populations. Relative sizes of immature DP and both mature CD4 and CD8 compartments were compared between 6-wk-old C57BL/ 6 mice ( $n=5$, black bars), transgenic C57BL/ 6 mice which express MHC class II I-E $\alpha^{\mathrm{d}}$ molecule in the thymus cortex (B6.tg E $\alpha^{\mathrm{d}}, n=4$; white bars) and B6.tgE $\alpha^{\mathrm{d}} \cdot \mathrm{A} \beta^{-/-}$mice ( $n=6$; gray bars) that only expresses the I-E molecule. Error bars represent standard deviation for each mouse group. Thymocyte subpopulations are expressed as percent of total thymocytes and were identified by flow cytometry using anti-mouse CD4, CD8 and TCR $\beta$ antibodies.
C57BL/6 strain (Fig. 6, 7). These results show that the lack of expression of I-E in the thymic cortex of C57BL/ 6 mice is responsible for the observed decrease in positive selection efficiency of CD4 single-positive cells.

The comparison of mice expressing only the I-A molecule with mice bearing both I-A and I-E molecules reveals that a sizable fraction of positively selected populations is attributable to selection by I-E (Fig. 7). At a lesser extent the same effect is observed in relation to the positively selected population attributable to the I-A molecule. In fact, the size of the positively selected populations in mice expressing either I-A or I-E is significantly smaller as compared to mice expressing both molecules in the thymic cortex, indicating that the number of expressed MHC class II genes controls the efficiency of positive selection. These results strongly suggest that the positively selected populations in mice bearing either I-A or I-E restriction elements do not contain a sizable fraction of TCR specificities that are represented in the selected repertoires of mice expressing both restriction elements.

Overall, when comparing mice with C57BL/6 background either or not expressing the MHC class II I-E molecule in different genetic models (congenics, bone marrow chimeras and transgenics), we noted an increase of about $50 \%$ in the size of the different DP populations expressing positive selection markers (Fig. 2, 4, 7). This implies that when the thymus simultaneously expresses I-A and I-E molecules, about $30 \%$ of the positively selected cells represent TCR specificities that exclusively recognize the I-E molecule. On the other hand, when the I-A molecule is introduced in mice transgenically expressing the I-E molecule, an increment in positive selection efficiency was obtained that ascribes $20 \%$ of the selected cells exclusively to the I-A molecule (Fig. 7). Together, the experiments reported here reveal the significance of the relative weight of gene-specific MHC class II restriction in the
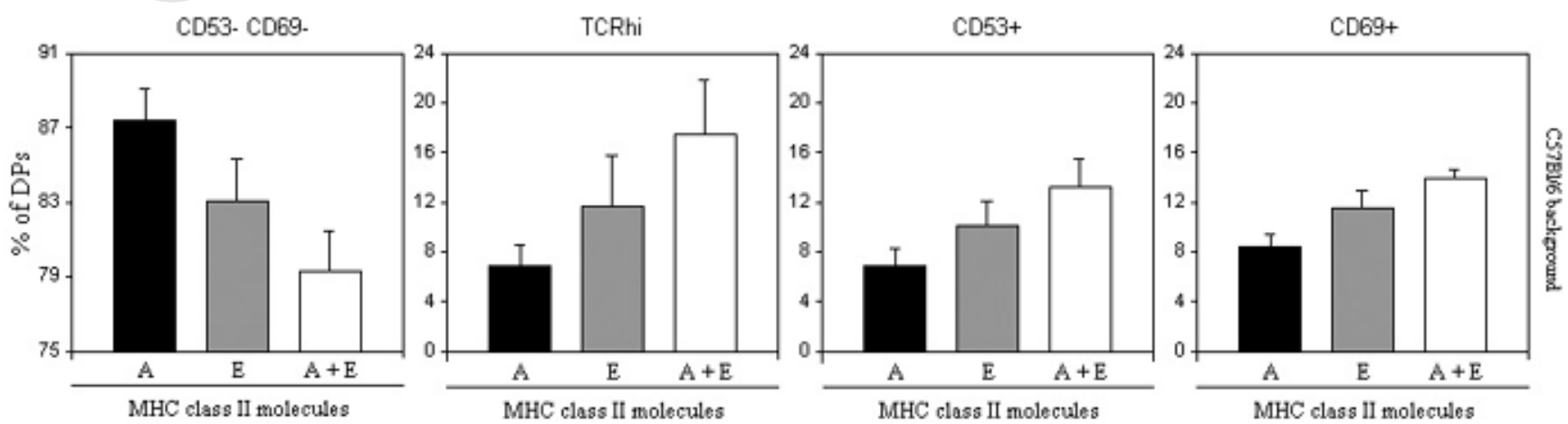

Figure 7. Multiple MHC class II gene expression in the thymic cortex controls the frequency of positively selected cells within the DP compartment. The relative sizes of thymocyte subpopulations within the immature DP compartment were measured in the mice represented in Fig. 5. Flow cytometry was performed using combinations of anti-mouse CD4 and CD8 with TCR, CD69 and CD53 antibodies. The error bars represent standard deviation for each mouse group. 
bulk thymic positive selection and bring attention to the contribution of MHC locus multigenic structure in increasing TCR repertoire diversity.

\section{Discussion}

Evaluation of the role of MHC molecules in the efficiency of positive selection has relied on the analysis of thymic selection in TCR transgenic models with defined specificities. In this study, we analyzed the role of MHC class II multiple genes in positive selection of thymocytes from naturally occurring pre-selection TCR repertoires. In different experimental settings, the results show that expression of MHC class II I-E molecule in the thymus is associated with higher efficiency of positive selection into the mature CD4 compartment as measured by the size of thymocyte subpopulations showing signs of positive selection.

However, the expression of the I-E molecule did not appear to reduce the efficiency of positive selection of CD8 mature T cells (data not shown). This observation is in line with other studies indicating that changing the efficiency of positive selection does not imply deviations in CD4/CD8 lineage commitment [20]. Moreover, we show that the differences in positive selection observed between mice carrying $\mathrm{H} 2^{\mathrm{b}}$ and $\mathrm{H} 2^{\mathrm{d}}$ haplotypes were not paralleled by quantitative differences in surface expression of MHC class II molecules on the thymic epithelium. In contrast with observations from some transgenic systems [11], these results suggest that the positive selection efficiency in non-manipulated mice may be controlled by mechanisms other than surface MHC class II density.

In fact, immunohistochemistry measurements of mice with different MHC class II genotypes showed that the density of MHC class II molecules on the thymic cortex was rather invariant. This indicates that the mechanisms that control the expression level of MHC class II molecules in the thymic cortex are not dependent on the MHC class II genotype and would not be accountable for the observed variation in positive selection efficiency. The experimental systems we analyzed are suggestive that MHC class II genetic configuration plays a role in positive selection efficiency, but we do not exclude that our results could also be influenced by other genes within the MHC haplotypes of the congenic strains or by MHC class II expression artefacts in transgenic strains.

Certain TCR specificities like 2B4 [21] and 5C.C7 [22] have proven to be selected only when the I-E molecule is expressed, but the relative abundance of such I-E molecule-restricted TCR reactivity among the selectable cells is unknown. Our results implicate the number of MHC class II functional loci in efficiency of thymic positive selection and raise the hypothesis that the repertoires of TCR specificities exclusively selected by either MHC class II I-A or I-E molecules represent a considerable fraction of the positively selected population. The presence of the I-E molecule accounts for around $50 \%$ increase in positive selection efficiency attributable to cells that would carry TCR specificities not selected by the I-A molecule. Conversely, the I-A molecule appears to increase the selected populations by $20 \%$ as compared to the efficiency of the I-E molecule alone.

This hypothesis may entail consequences in regard to current views on the role of MHC-TCR affinity/avidity in repertoire selection [23-25]. Particularly, it would imply that the range of affinities within the positive selection window of one MHC class II gene would be narrow, as it would not target a set of TCR specificities selectable by the other MHC class II gene. This could be seen as a mechanism to warrant that the mature TCR repertoires selected by these two molecules contain a sizable proportion of non-redundant TCR specificities. In such case, the MHC locus multigenic structure could serve to increase TCR repertoire diversity by allowing the selection of a large number of MHC gene-restricted TCR specificities that conceivably could play different functional roles.

In this regard, it is interesting to note that mutations abrogating E molecule expression are relatively frequent both in wild-derived and inbred mouse strains [26], while A molecule null mutants have not been found, suggesting that E molecule expression is not under high selective pressure. Nevertheless, absence of E molecule expression has been correlated with MHC haplotypes that confer susceptibility to autoimmune diseases [27, 28]. The role of E molecule absence in pathogenesis of autoimmunity has been addressed in complementation experiments with E molecule transgenes [29, 30], but evidence that endogenous E molecule expression protects from autoimnunity has not yet been provided.

On the other hand, it is well known that MHC locus polymorphism contributes to the diversity of the selected TCR repertoire. However, $\mathrm{MHC}^{\mathrm{b} / \mathrm{d}} \mathrm{F} 1$ mice expressing two different alleles of the I-A molecule did not show a measurable increase in positive selection efficiency as compared to $\mathrm{MHC}^{\mathrm{d}}$ homozygous mice. We speculate that the increase in repertoire diversity generated by one allele of the I-A molecule may occur at the expenses of the efficiency of the other allele.

Alternative models of thymic selection have been proposed that are based on dual recognition of TCRMHC binding. According to such models the TCR contact with MHC is functionally distinct from the contact with the peptide as opposed to the current models of context recognition of the MHC plus peptide. Our observations 
implying that different MHC class II genes may select an exclusive repertoire of TCR specificities, are compatible with the hypothesis entailed by some dual recognition models [31, 32] claiming that MHC class II molecules encode invariant restricting elements for TCR binding. In this frame, our results would suggest that invariant restricting elements are gene-specific (but allelic invariant), which may imply gene-specific constraints and requirements for TCR recognition.

Independently of the molecular mechanisms that may be underlying selection of gene-specific TCR specificities, if increased efficiency of positive selection would imply acquisition of MHC gene-related functions by the TCR repertoire, then the presence of multiple genes in each MHC class would confer an evolutionary advantage. In this context, it is striking that the multigenic structure of MHC class II genes is maintained to code for two to three molecules per individual across phylogeny from fish to man [33], which could represent an equilibrium between maintaining a coherent selfrecognizable immune system and extending the TCR repertoire functions.

\section{Materials and methods}

\section{Mice}

Mice were bred and maintained in conventional housing facilities at the Instituto Gulbenkian de Ciência and Table 1 shows the list of the different mouse strains analyzed in this study. All mice analyzed in this study were females. B6.C$\mathrm{H} 2<\mathrm{d}>/ \mathrm{bBy}$ is a congenic strain carrying the $\mathrm{MHC}^{\mathrm{d}}$ haplotype in the C57BL/ 6 genetic background (the congenic interval is comprised between marker D17MIT198 and D17MIT152). C.B10-H2 $<\mathrm{b}>/$ LilMcd is a congenic strain carrying the $\mathrm{MHC}^{\mathrm{b}}$ haplotype in the $\mathrm{BALB} / \mathrm{c}$ genetic background (the congenic interval is comprised between marker D17MIT80 and D17MIT232). Both strains were purchased from the Jackson Laboratory (Bar Harbor, ME). The F1 progeny of C57BL/ 6 and B6.C-H2 $<\mathrm{d}>/$ bBy mice was designated B6.C-H2 $<$ bd $>$ and the $\mathrm{F} 1$ progeny of BALB/c and C.B10-H $2<\mathrm{b}>/$ LilMcd mice was named C.B10-H2 $<$ bd $>$.

Lymphocyte-deficient mice carrying the $\mathrm{H} 2^{\mathrm{d}}$ haplotype in homozygous state were obtained by crossing the B6.Rag2 $2^{-/-}$ mouse strain (originally from CDTA, Orléans, France) with the B6.C-H2 $<\mathrm{d}>/$ bBy strain. The resulting mice have been named B6.Rag2 ${ }^{-/-}$.C-H $2<\mathrm{d}>$. The $\mathrm{F} 1$ progeny originated from crossing these mice with B6.Rag $2^{-/-}$mice was named B6.Rag $2^{-/-}$.C$\mathrm{H} 2<\mathrm{bd}>$.

Transgenic mice expressing MHC class II E $\alpha^{\mathrm{d}}$ in the thymic epithelia and mice that in addition to the $\mathrm{Ea}^{\mathrm{d}}$ transgene carry a MHC class II $A \beta^{\mathrm{b}}$ knockout mutation have been described previously [18, 19, 34] and were a kind gift from Dr. Antonio Bandeira at the Institut Pasteur (Paris, France). These strains are here referred to as B6.tg $\mathrm{E} \alpha^{\mathrm{d}}$ and B6.tg $\mathrm{E} \alpha^{\mathrm{d}} . \mathrm{A} \beta^{-/-}$, respectively. The B6.tg $\mathrm{E} \alpha^{\mathrm{d}} . \mathrm{A} \beta^{-/-}$strain was obtained from backcrossing the $A \beta^{-/-}$mutation into the $\mathrm{C} 57 \mathrm{BL} / 6$ genetic background for eight generations [35] followed by crossing the $\mathrm{E} \alpha \mathrm{d}$ transgene. The MHC locus of this strain was extensively genotyped as haplotype b from marker D17MIT62 to D17MIT232. The B6.tg $\mathrm{E} \alpha^{\mathrm{d}}$ mice used in this study were obtained by further backcrossing into the C57BL/ 6 genetic background for three generations. Nude mice were originally purchased as the B6.Cg/Ntac-Foxn $1^{\text {nu }}$ strain from Jackson Laboratory and were bred in our animal facilities. All procedures were in accordance with national regulations on animal experimentation and welfare.

\section{Flow cytometry}

Five-week-old female mice were sacrificed, their thymii collected and single-cell suspensions were prepared by gently squeezing the organs through a nylon mesh. Cell surface fourcolor staining was performed using the following monoclonal antibody (mAb) combinations: anti-CD53 (OX-80; Serotec, Kidlington, UK) plus FITC-coupled anti-rat IgM (MARM-4, Serotec) or FITC-coupled anti-TCR $\beta$ (H57-597), PE-coupled anti-CD69 (H1.2F3; Becton Dickinson), biotin coupled antiCD4 (L3T4/RM4-5; BD Pharmingen, San Diego, CA) plus PerCP-coupled streptavidin (BD Pharmingen), allophycocyanin-coupled anti-CD8 $\alpha$ (YTS169.4). Sample acquisition was performed using a FACSCalibur ${ }^{\mathrm{TM}}$ (BD Pharmingen) and analysis used the FlowJo software (Tree Star, Inc., Ashland, OR).

\section{Bone marrow cell transfers}

Donor bone marrow cells were harvested from flushed marrow cavities from femurs and tibiae of nude mice. Recipient mice at 7 wk of age were lethally irradiated with 7.5 Gy using a ${ }^{137} \mathrm{Cs}$ source and then intravenously injected with approximately 4.1 million bone marrow donor cells. Recipients comprised three different genotype groups: B6.Rag2 $2^{-/-}$, B6.Rag2 $2^{-/-}$.C$\mathrm{H} 2<\mathrm{bd}>$ and B6.Rag2 ${ }^{-/}$.C-H2 $<\mathrm{d}>$. Fifteen weeks after transfer peripheral blood staining for CD4 and CD8 was performed to confirm $\mathrm{T}$ cell compartment reconstitution after which the mice were killed and thymocytes analyzed by flow cytometry.

\section{Immunohistochemistry}

Five- to six-week-old female mice were sacrificed and their thymii were collected, immersed in Tissue Tek $^{\circledR}$ O.C.T. ${ }^{\mathrm{TM}}$ Compound (Sakura Finetek, Zoeterwoude, The Netherlands) and stored at $-20^{\circ} \mathrm{C}$. For each frozen thymus two to three $8-\mu \mathrm{m}$ sections were obtained and processed for immunostaining. Sections were immersed in cold acetone for $10 \mathrm{~min}$ and stored at $-20^{\circ} \mathrm{C}$. For staining, the sections were covered with PBS with $3 \%$ FCS for 45 min and were incubated with biotinylated antimouse MHC class II mAb (clone M5/114) for $45 \mathrm{~min}$. This was followed by incubation with avidin-rhodamine for $30 \mathrm{~min}$ in dark closed moist atmosphere chamber. Glass slides were mounted in Fluoromount-G (Southern Biotechnology Associates, Inc., Birmingham, AL) with cover slides. Sections were analyzed with a Leica DMRA2 microscope equipped with a cooled CCD camera. Photographs of both cortex and medulla were taken as 12-bit images using $20 \times$ magnification. Fluorescence intensity was measured using ImageJ software. 
Individual fluorescence measurements were taken by manually selecting defined cortex and medulla areas. For flat field correction the detection system was calibrated with a fluorescent slide. For each slide the level of minimum fluorescence (background) was determined in areas with no tissue and subtracted to mean fluorescence software calculated values. In the cortex of C57BL/6, B6.C-H2 $<\mathrm{d}>/$ bBy and BALB/ c mice a total of 7,11 and 12 fields were observed and 11, 22 and 24 areas for fluorescence measurements were selected, respectively. Likewise, 6, 13 and 12 fields were observed and 11, 22 and 24 fluorescence measurements were taken for the medulla. Mean fluorescence intensities are reported as normalized cortex/medulla ratios that were calculated in each mouse by taking the average of the mean fluorescence intensity of the measured cortex fields over the average of the mean fluorescence intensity of the measured medulla fields. Cortex/medulla ratios in each strain represent the average of the ratios of individual animals.

Acknowledgements: We wish to thank Dr. António Bandeira for making available to us the MHC classII A $\beta$ null mice and transgenic mice expressing MHC classII Ead, and Nuno Moreno for technical help in immunohistochemistry measurements. We are grateful to Drs. Antonio Coutinho, Jocelyne Demengeot and Melvin Cohn for suggestions and insightful comments on the manuscript.

\section{References}

1 von Boehmer, H., Aifantis, I., Gounari, F., Azogui, O., Haughn, L., Apostolou, I., Jaeckel, E. et al., Thymic selection revisited: How essential is it? Immunol. Rev. 2003. 191: 62-78.

2 Starr, T. K., Jameson, S. C. and Hogquist, K. A., Positive and negative selection of T cells. Annu. Rev. Immunol. 2003. 21: 139-176.

3 Wong, P., Goldrath, A. W. and Rudensky, A. Y., Competition for specific intrathymic ligands limits positive selection in a TCR transgenic model of $\mathrm{CD}^{+}{ }^{+} \mathrm{T}$ cell development. J. Immunol. 2000. 164: 6252-6259.

4 Sakaguchi, N., Takahashi, T., Hata, H., Nomura, T., Tagami, T., Yamazaki, S., Sakihama, T. et al., Altered thymic T-cell selection due to a mutation of the ZAP-70 gene causes autoimmune arthritis in mice. Nature 2003. 426: 454-460.

5 Barton, G. M. and Rudensky, A. Y., Evaluating peptide repertoires within the context of thymocyte development. Semin. Immunol. 1999. 11: 417-422.

6 Faro, J., Velasco, S., Gonzalez-Fernandez, A. and Bandeira, A., The impact of thymic antigen diversity on the size of the selected T cell repertoire. J. Immunol. 2004. 172: 2247-2255.

7 Chmielowski, B., Muranski, P. and Ignatowicz, L., In the normal repertoire of $\mathrm{CD}^{+}{ }^{+} \mathrm{T}$ cells, a single class II MHC/peptide complex positively selects TCRs with various antigen specificities. J. Immunol. 1999. 162: 95-105.

8 Zinkernagel, R. M. and Althage, A., On the role of thymic epithelium $v s$. bone marrow-derived cells in repertoire selection of T cells. Proc. Natl. Acad. Sci. USA 1999. 96: 8092-8097.

9 Zerrahn, J., Volkmann, A., Coles, M. C., Held, W., Lemonnier, F. A. and Raulet, D. H., Class I MHC molecules on hematopoietic cells can support intrathymic positive selection of T cell receptor transgenic T cells. Proc. Natl. Acad. Sci. USA 1999. 96: 11470-11475

10 Ashton-Rickardt, P. G., Bandeira, A., Delaney, J. R., Van Kaer, L., Pircher, H. P., Zinkernagel, R. M. and Tonegawa, S., Evidence for a differential avidity model of T cell selection in the thymus. Cell 1994. 76: 651-663.
11 Martinic, M. M., Rocha, B., McCoy, K. D., Hengartner, H. and Zinkernagel, R. M., Role of TCR-restricting MHC density and thymic environment on selection and survival of cells expressing low-affinity T cell receptors. Eur. J. Immunol. 2004. 34: 1041-1049.

12 Wong, F. S. and Wen, L., What can the HLA transgenic mouse tell us about autoimmune diabetes? Diabetologia 2004. 47: 1476-1487.

13 Robey, E. A. and Bousso, P., Visualizing thymocyte motility using 2-photon microscopy. Immunol. Rev. 2003. 195: 51-57.

14 Zinkernagel, R. M., Pircher, H. P., Ohashi, P., Oehen, S., Odermatt, B., Mak, T., Arnheiter, H. et al., T and B cell tolerance and responses to viral antigens in transgenic mice: Implications for the pathogenesis of autoimmune versus immunopathological disease. Immunol. Rev. 1991. 122: $133-171$

15 Duarte, N. and Penha-Goncalves, C., The MHC locus controls size variations in the CD4 compartment of the mouse thymus. Immunogenetics 2001. 53: 662-668.

16 Tomlinson, M. G., Hanke, T., Hughes, D. A., Barclay, A. N., Scholl, E., Hunig, T. and Wright, M. D., Characterization of mouse CD53: Epitope mapping, cellular distribution and induction by $\mathrm{T}$ cell receptor engagement during repertoire selection. Eur. J. Immunol. 1995. 25: 2201-2205.

17 Puls, K. L., Hogquist, K. A., Reilly, N. and Wright, M. D., CD53, a thymocyte selection marker whose induction requires a lower affinity TCRMHC interaction than CD69, but is up-regulated with slower kinetics. Int. Immunol. 2002. 14: 249-258.

18 Widera, G., Burkly, L. C., Pinkert, C. A., Bottger, E. C., Cowing, C., Palmiter, R. D., Brinster, R. L. and Flavell, R. A., Transgenic mice selectively lacking MHC class II (I-E) antigen expression on B cells: An in vivo approach to investigate Ia gene function. Cell 1987. 51: 175-187.

19 Brocker, T., Survival of mature CD4 T lymphocytes is dependent on major histocompatibility complex class II-expressing dendritic cells. J. Exp. Med. 1997. 186: 1223-1232.

20 Lucas, J. A., Atherly, L. O. and Berg, L. J., The absence of Itk inhibits positive selection without changing lineage commitment. J. Immunol. 2002. 168: 6142-6151.

21 Berg, L. J., Pullen, A. M., Fazekas de St Groth, B., Mathis, D., Benoist, C. and Davis, M. M., Antigen/MHC-specific T cells are preferentially exported from the thymus in the presence of their MHC ligand. Cell 1989. 58: 1035-1046.

22 Seder, R. A., Paul, W. E., Davis, M. M. and Fazekas de St Groth, B., The presence of interleukin 4 during in vitro priming determines the lymphokine-producing potential of $\mathrm{CD}^{+} \mathrm{T}$ cells from $\mathrm{T}$ cell receptor transgenic mice. J. Exp. Med. 1992. 176: 1091-1098.

23 Davis, M. M. and Bjorkman, P. J., T-cell antigen receptor genes and T-cell recognition. Nature 1988. 334: 395-402.

24 Matzinger, P., Why positive selection? Immunol. Rev. 1993. 135: 81-117.

25 Janeway, C. A., Jr., Dianzani, U., Portoles, P., Rath, S., Reich, E. P., Rojo, J., Yagi, J. and Murphy, D. B., Cross-linking and conformational change in T-cell receptors: Role in activation and in repertoire selection. Cold Spring Harb. Symp. Quant. Biol. 1989. 54: 657-666.

26 Tacchini-Cottier, F., Mayer, W. E., Begovich, A. B. and Jones, P. P., Inactivation of $\mathrm{E}$ alpha and $\mathrm{E}$ beta expression in inbred and wild mice by multiple distinct mutations, some of which predate speciation within Mus species. Int. Immunol. 1995. 7: 1459-1471.

27 Brand, D. D., Whittington, K. B. and Rosloniec, E. F., I-Aq and I-Ap bind and present similar antigenic peptides despite differing in their ability to mediate susceptibility to autoimmune arthritis. Autoimmunity 2001. 34: 133-145.

28 Hattori, M., Buse, J. B., Jackson, R. A., Glimcher, L., Dorf, M. E., Minami, M., Makino, S. et al., The NOD mouse: Recessive diabetogenic gene in the major histocompatibility complex. Science 1986. 231: 733-735.

29 Bohme, J., Schuhbaur, B., Kanagawa, O., Benoist, C. and Mathis, D., MHC-linked protection from diabetes dissociated from clonal deletion of T cells. Science 1990. 249: 293-295.

30 Lund, T., O'Reilly, L., Hutchings, P., Kanagawa, O., Simpson, E., Gravely, R., Chandler, P. et al., Prevention of insulin-dependent diabetes mellitus in non-obese diabetic mice by transgenes encoding modified I-A beta-chain or normal I-E alpha-chain. Nature 1990. 345: 727-729. 
31 Cohn, M., Tritope model of restrictive recognition by the TCR. Trends Immunol. 2003. 24: 127-131.

32 Cohn, M., An alternative to current thinking about positive selection, negative selection and activation of T cells. Immunology 2004. 111: 375-380.

33 Kelley, J., Walter, L. and Trowsdale, J., Comparative genomics of major histocompatibility complexes. Immunogenetics 2005. 56: 683-695.
34 Pimenta-Araujo, R., Mascarell, L., Huesca, M., Cumano, A. and Bandeira, A., Embryonic thymic epithelium naturally devoid of APCs is acutely rejected in the absence of indirect recognition. J. Immunol. 2001. 167: 5034-5041.

35 Lee, R. S., Grusby, M. J., Laufer, T. M., Colvin, R., Glimcher, L. H. and Auchincloss, H., Jr., CD8 ${ }^{+}$effector cells responding to residual class I antigens, with help from $\mathrm{CD} 4^{+}$cells stimulated indirectly, cause rejection of "major histocompatibility complex-deficient" skin grafts. Transplantation 1997. 63: 1123-1133 